

Brazilian Journal of Physics

ISSN: 0103-9733

luizno.bjp@gmail.com

Sociedade Brasileira de Física

Brasil

Jafari, A.; Mabhouti, KH.; Heydari Heydarabad, M.

Effect of the External Mirror Feedback Strength in the Dynamics and Spectrum of the Injected

Semiconductor Lasers

Brazilian Journal of Physics, vol. 44, núm. 1, 2014, pp. 8-18

Sociedade Brasileira de Física

Sâo Paulo, Brasil

Available in: http://www.redalyc.org/articulo.oa?id=46429745002

- How to cite

Complete issue

- More information about this article

Journal's homepage in redalyc.org

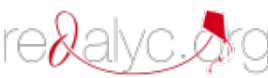

Scientific Information System

Network of Scientific Journals from Latin America, the Caribbean, Spain and Portugal

Non-profit academic project, developed under the open access initiative 


\title{
Effect of the External Mirror Feedback Strength in the Dynamics and Spectrum of the Injected Semiconductor Lasers
}

\author{
A. Jafari • KH. Mabhouti • M. Heydari Heydarabad
}

Received: 9 April 2012 /Published online: 20 December 2013

(C) Sociedade Brasileira de Física 2013

\begin{abstract}
We analytically investigate the effect of an external mirror on the stability of an injected semiconductor laser, the latter treated as injected damped oscillators. In the studied configuration, the injected semiconductor laser with an external mirror is under the influence of a chaotic oscillating optical signal that is generated by a similar semiconductor master laser. We derive our results in terms of the damping rate and resonance frequency. We show that the external mirror can eliminate the unstable modes of the injected laser at low frequencies. Furthermore, the mirror can enhance the damping of the oscillation modes of the injected semiconductor laser; consequently, the driven response of the injected laser may have a broad spectrum, even wider than that of the chaotic driving signal. We also show results for the bandwidths of the injection amplitude and phase increment as functions of the injection rate and feedback strength of the external mirror. In addition, we use bifurcation and time-series curves to describe the dynamical behavior of the injected laser. We identify the feedback strength of injected laser, relative to that of the master laser, which induces synchronization between the injected-laser oscillation modes and the master laser.
\end{abstract}

Keywords Semiconductor lasers $\cdot$ Synchronization . Bifurcation

\section{Introduction}

Optical injection is a fundamental technique for coupling or controlling injected slave lasers to the output of master lasers. As such, injecting phenomena have been intensively studied

\footnotetext{
A. Jafari $(\bowtie) \cdot$ K. Mabhouti $\cdot$ M. H. Heydarabad

Physics Department, Faculty of Sciences, Urmia Uni'versity,

PO Box 165, Urmia, Iran

e-mail: a.jafari@urmia.ac.ir
}

for the synchronization of optical oscillations, stabilization of lasers, noise reduction, enhancement on modulation bandwidth, and reduction of the chirp-to-power ratio [1-3].

Semiconductor lasers subject to optical feedback from external mirrors or optical injection from other lasers are nonlinear optical systems that can generate chaotic intensity fluctuations. Semiconductor lasers are the most important source generators in secure optical communications, coherent light sources for technological optical transition, and ultra-fast optical processing [4-6]. For this reason, the dynamics of semiconductor lasers with optical feedback is currently the subject of experimental and theoretical investigations [7].

Communication via chaotic signals has drawn much attention in the last two decades. Chaos synchronization of nonlinear oscillators has been widely studied for applications in secure communications [8]. A semiconductor laser with optical feedback exhibits chaotic dynamics owing to the infinite degrees of freedom generated by delayed feedback and is therefore an excellent nonlinear oscillator [9, 10]. A semiconductor laser subject to an external feedback displays rich nonlinear dynamics including quasiperiodicity, regular pulse package, and chaos [11, 12].

Systems of two chaotic lasers have been shown to synchronize with each other and are excellent candidates for fast and secure communication [13-15]. The optical injection can be used for two purposes. First, the injection signal can induce such instabilities as periodic and the chaotic behaviors. Second, it can also be used as an effective mean to improve the frequency stability, enhance the bandwidth, and reduce the noise and distortion characteristics $[1,3,16]$.

Successful chaos-based communication, supported by the synchronization of an emitter master laser (ML) with a receiver slave laser (SL), is achieved by injecting part of the light emitted by the ML into the SL. The dynamical behavior of the SL injected by the ML can be affected by different parameters, such as the feedback phase, feedback strength, and injection rate. 
In [17], the dynamical response of a slave semiconductor laser, with short cavity, under the injection of chaotic light from a master semiconductor laser, was widely studied. The phase and strength of the feedback parameters were shown to affect the dynamical response of the slave laser. Our numerical calculations showed that the dynamics of the slave laser may imitate the master laser, depending on the feedback strength. It also heavily depends on the dynamics of the injection field. In this paper, to study the influence of an external mirror (EM) upon the SL dynamical properties, we consider the appropriate values of the injection rate determined in [17] and generalize the analysis in [18] for unidirectional coupled optical setup for the dimensionless rate equations. The generalization enables us to extend and complete the report of our work in [17] with more details, such as stability, bandwidth, amplitude, and frequency of the SL.

The injection laser is always subject to a constant-intensity bias-field injection in the absence of the driving signal. This bias-field injection can induce major changes in the damping properties of the laser, regarded as a set of damped oscillators. On the basis of an analysis considering the injected laser as one such set of driven damped oscillators, we have found the EM to have important effects on the SL, beyond chaos synchronization. We have verified that the addition of an EM to the SL may not only substantially affect the stability of the SL but also synchronize its dynamical behavior to that of the ML, provided that the relative feedback strength of the SL be appropriately selected with respect to the ML. These conclusions are drawn from an analysis of bifurcation and time-series curves.

This paper is organized as follows. In Section 2, we describe the setup of the experimental system. In Section 3, we perform a linear stability analysis for the rate equations and numerically express the results. In Section 4, we investigate the effect of an EM on the stability, bandwidth, amplitude, and frequency of resonance of the SL. In Section 5, we study the stability of the SL output intensity $\left|E_{\mathrm{s}}\right|^{2}$ as a function of the changes of the feedback phase by bifurcation and time series. Finally, Section 6 collects our conclusions.

\section{Model and Rate Equations}

The optical setup for chaos synchronization is shown in Fig. 1. The master and slave systems are identical. The slave system comprises a semiconductor laser and external mirror.

The master chaotic output is unidirectionally optically injected into the active layer of the slave laser. Semiconductor lasers can usually be successfully modeled by two dynamical equations, one for the evolution of the slowly varying complex electric field amplitude $E(t)$, the other for the evolution of the carrier population $N(t)$. Particularly valuable for our purposes are the Lang and Kobayashi equalities, which are the usual laser rate equations

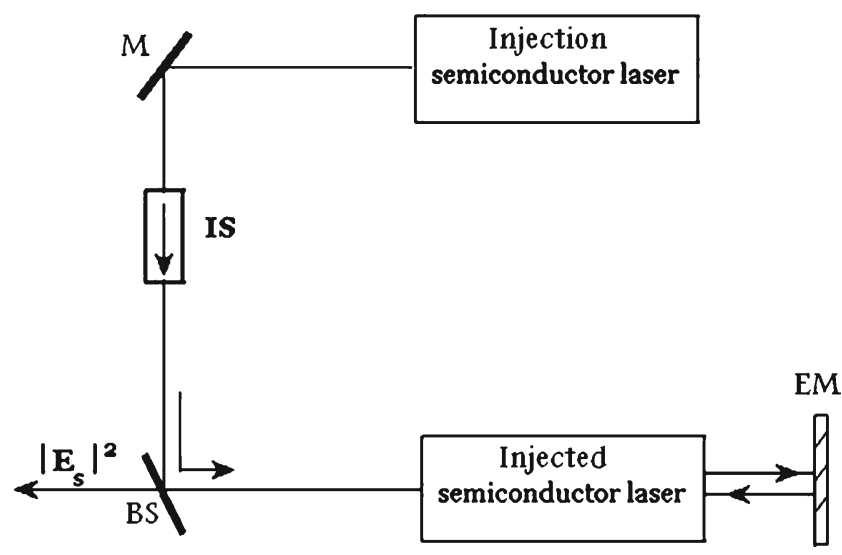

Fig. 1 Optical setup. $I S$ isolator, $E M$ external mirror, $M$ mirror, $B S$ beam splitter

plus a time-delayed term taking the field reflected from the EM into account,

$$
\begin{aligned}
& \frac{\mathrm{d} E_{\mathrm{m}}}{\mathrm{d} t}=(1+i \alpha) N E_{\mathrm{m}}+\eta E_{\mathrm{m}} e^{-i C_{\mathrm{p}}} \\
& \frac{\mathrm{d} E_{\mathrm{s}}}{\mathrm{d} t}=(1+i \alpha) N E_{\mathrm{s}}+k E_{\mathrm{s}}(t-\tau) e^{-i C_{\mathrm{p}}}+\beta E_{\mathrm{m}}(t) \\
& T \frac{\mathrm{d} N}{\mathrm{~d} t}=P-N-(1+2 N)\left|E_{\mathrm{m}, \mathrm{s}}\right|^{2},
\end{aligned}
$$

where the subscripts $m$ and $s$ distinguish the complex electric fields $E_{\mathrm{m}}$ and $E_{\mathrm{s}}$, in the master and the slave lasers, respectively. $N$ is the inversion parameter, $\alpha$ is the line width enhancement factor, and $\eta$ and $k$ are the feedback strengths of the ML and of the EM associated with the SL, respectively. $C_{\mathrm{p}}$ is the $2 \pi$-periodic feedback phase, and $P$ is the pump current. In these equations, the time is normalized to the cavity photon lifetime ( $1 \mathrm{ps}$ ), and $T$ is the ratio of the carrier lifetime (1 ns) to the photon lifetime [19]. The external round trip time $\tau$ is also normalized to the photon lifetime. $\left|E_{\mathrm{m}}\right|^{2}$ is the amplitude of the injection light and the injection strength is $\beta$. Four of the parameters are held fixed at $T=1710, P=0.8$, $\tau=70$, and $\alpha=5$ [20].

In our study, a feedback-induced chaos is injected from the ML into the SL, which contains an EM. We perform a linear stability analysis for the rate equations and analytically investigate the bandwidth of the optically injected semiconductor laser. Equation 1 has only been spelled out for completeness and will play no role in the following analysis.

\section{Linear Stability Analysis}

The most common model for injection-locked lasers uses a set of three differential equations [3,21]. The differential equation 
governing the complex field of an injection-locked laser is similar to that of a free-running laser, with the addition of an injection term,

$$
\frac{\mathrm{d} E(t)}{\mathrm{d} t}=(1+i \alpha) N E(t)+k E(t-\tau) e^{-i C_{\mathrm{p}}}+\beta E_{\mathrm{inj}}
$$

where $E(\mathrm{t})$ is the SL complex field, and the coupling term is $E_{\text {inj }}(\mathrm{t})=E_{\mathrm{m}}(t)$.

Here, for increased numerical accuracy, we have used the dimensionless form of the Lang-Kobayashi equations [20, 22].

This model ignores spontaneous emission and noise. Since chaotic electric fields emitted from semiconductor lasers are usually modulated in both amplitude and phase, it is appropriate to assume the injection field, as well as slave-laser field, to be modulated, as in the expression $E_{\text {inj }}(t)=A_{\text {inj }}(t) \exp \left[i \phi_{\text {inj }}(\mathrm{t})\right]$. The resulting split equations, along with the carrier-rate equation, constitute the following set of three differential equations describing injectionlocked lasers [3]:

$$
\begin{gathered}
\frac{\mathrm{d} A(t)}{\mathrm{d} t}=N(t) A(t)+k A(t-\tau) \cos \left[\phi(t)-\phi(t-\tau)+C_{\mathrm{p}}\right]+ \\
\beta A_{\text {inj }}(t) \cos \left[\phi(t)-\phi_{\text {inj }}(t)\right]
\end{gathered}
$$

$$
\begin{gathered}
\frac{\mathrm{d} \phi(t)}{\mathrm{d} t}=\alpha N(t)-k \frac{A(t-\tau)}{A(t)} \sin \left[\phi(t)-\phi(t-\tau)+C_{\mathrm{p}}\right]- \\
\beta \frac{A_{\mathrm{inj}}(t)}{A(t)} \sin \left[\phi(t)-\phi_{\mathrm{inj}}(t)\right]
\end{gathered}
$$

$T \frac{\mathrm{d} N}{\mathrm{~d} t}=P-N-(1+2 N)|E|^{2}$

where $A(t), \phi(t)$, and $N(t)$ are the SL field magnitude, field phase and carrier number. In these equations, $\phi(t)=\phi(t-\tau)-$ $C_{\mathrm{p}}(\bmod 2 \pi)[23]$.

Due to the nature of optical injection, a signal injects the laser on a dc bias component of the injected field. Therefore, we have divided the injecting-field amplitude into time-constant and time-varying terms and analyzed Eqs. (5), (6), and (7), including the signal term, and derived the injection response of the injected laser around the stationary state in two steps. In the first step, we have investigated the damping oscillation properties of the laser with no signal, i.e., with the timevarying terms set to zero: $a_{i n j}(t)=\phi_{i n j}(t)=0$. In the second step, we have assumed the signal term to have the perturbation form $A_{\text {inj }}(t)=A_{\text {inj,c }}+\delta a_{\text {inj }} \exp (\lambda t)$ and $\phi_{\text {inj }}(t)=\phi_{\text {inj,c }}+\delta \phi_{\text {inj }} \exp (\lambda t)$, like the other variables.

In the first step, we assumed the injected field to have the constant amplitude and phase $A_{\text {inj }}(t)=A_{\text {inj,c }}$ and $\phi_{\text {inj }}(t)=\phi_{\text {inj,c }}$, respectively. From Eqs. 5, 6, and 7, the stationary solutions $A(t)=A_{\mathrm{st}}, \phi(t)=\phi_{\mathrm{st}}$ and $N(t)=N_{\mathrm{st}}$ are then given by the expressions

$A_{\mathrm{st}}^{2}=\frac{P-N_{\mathrm{st}}}{1+2 N_{\mathrm{st}}}$

$\Delta \varphi_{s t}=\varphi_{\mathrm{st}}-\varphi_{\mathrm{inj, \textrm {c }}}=-\arctan \alpha+\arcsin \left(\frac{-\alpha k}{\beta \sqrt{1+\alpha^{2}}}\right)$

$N_{\mathrm{st}}=-k-\beta \cos \Delta \phi_{\mathrm{st}}$

Equation (10) is a simplified form of $N_{\mathrm{st}}=-k \cos \theta$ $-\beta \cos \Delta \phi_{\mathrm{st}}$, where $\theta=\phi(t)-\phi(t-\tau)+C_{\mathrm{p}},(-\pi \leq \theta \leq+\pi)$. Here, for simplicity, we have chosen $\theta=0$.

Changes in $C_{\mathrm{p}}$ for the EM qualitatively affect the SL dynamics. For specific values of $k$ and $C_{\mathrm{p}}$, the dynamics include a set of periodic, quasi-periodic, chaotic, and regular pulse-package behaviors. Accordingly, $C_{\mathrm{p}}$ can be tuned to make the operation of the laser stable or unstable. On the other hand, with an appropriate choice of $\beta$, the injected beam can change the SL dynamics to follow the ML dynamics [17].

As explained in [18], we let $A_{\mathrm{inj,c}}=A_{\text {st }}$ for the injected field to simplify the analysis. Next, we consider a small perturbation in the form $x(t)=x_{\mathrm{st}}+\delta x \exp (\lambda t)(x=A, \phi$, $N)$, where we have introduced the complex perturbation parameter $\lambda$ to study the stability of the injected laser. After inserting the small perturbation and the stationary condition into the rate equations and linearizing them, we obtain the following equations for the perturbations in matrix form:

$\left[\begin{array}{lcc}\lambda-N_{s t} & \alpha N_{\mathrm{st}} A_{\mathrm{st}} & -A_{\mathrm{st}} \\ -\alpha \frac{N_{s t}}{A_{s t}} & \lambda-N_{\mathrm{st}} & -\alpha \\ 2 T^{-1}\left(1+2 N_{s t}\right) A_{\mathrm{st}} & 0 & \lambda+T^{-1}\left(1+2 A_{\mathrm{st}}^{2}\right)\end{array}\right]\left[\begin{array}{l}\delta A \\ \delta \phi \\ \delta N\end{array}\right]=0$

The determinant of the coefficient matrix is given by the equality

$$
\begin{gathered}
D(\lambda)=\lambda^{3}+\left[\varepsilon\left(1+2 A_{\mathrm{st}}^{2}\right)-2 N_{\mathrm{st}}\right] \lambda^{2}+\left[\left(1+\alpha^{2}\right) N_{\mathrm{st}}^{2}-\right. \\
\left.2 \varepsilon\left(1+2 A_{\mathrm{st}}^{2}\right) N_{\mathrm{st}}+2 \varepsilon\left(1+2 N_{\mathrm{st}}\right) A_{\mathrm{st}}^{2}\right] \lambda+ \\
\left(1+\alpha^{2}\right) \varepsilon N_{\mathrm{st}}^{2}\left(1+2 A_{\mathrm{st}}^{2}\right)-2 N_{\mathrm{st}} \varepsilon\left(1+2 N_{\mathrm{st}}\right) A_{\mathrm{st}}^{2}\left(1+\alpha^{2}\right),
\end{gathered}
$$


where $\varepsilon=\frac{1}{T}$ and we have fixed the parameters $T$ and $\alpha$ at 1,710 and 5 , respectively.

The injected laser will only be stable if the determinant in Eq. 12 vanishes. To make the right-hand side equal to zero, we have to adjust the complex perturbation parameter $\lambda=\gamma+i w$, in which $\gamma<0$ corresponds to damped oscillation modes, while $\gamma>0$ corresponds to unstable oscillation modes. The imaginary part $w$ is the angular frequency of the damping oscillation $[24,25]$.

In the second step of our procedure, insertion of the signal terms with all the other perturbed variables into Eqs. (5), (6), and (7) leads to the following set of linear equations:

$$
\begin{gathered}
\lambda \delta A=N_{\mathrm{st}} \delta \mathrm{A}+A_{\mathrm{st}} \delta N-\beta A_{\mathrm{st}} \sin \Delta \varphi_{\mathrm{st}}\left(\delta \phi-\delta \phi_{\mathrm{inj}}\right) \\
+\beta \cos \Delta \phi_{\mathrm{st}} \delta a_{\mathrm{inj}}
\end{gathered}
$$

$$
\begin{aligned}
\lambda \delta \phi=\alpha \delta N-k \delta \phi+ & \frac{\beta \sin \Delta \phi_{\mathrm{st}}}{A_{\mathrm{st}}} \delta A-\beta \cos \Delta \phi_{\mathrm{st}}\left(\delta \phi-\delta \phi_{\mathrm{inj}}\right) \\
& -\beta \frac{\sin \Delta \phi_{\mathrm{st}}}{A_{\mathrm{st}}} \delta \alpha_{\mathrm{inj}}
\end{aligned}
$$

$T \lambda \delta N=-\delta N-2 A_{\mathrm{st}}^{2} \delta N-2 A_{\mathrm{st}}\left(1+2 N_{\mathrm{st}}\right) \delta A$
Fig. 2 Damping rate $\gamma$ of the oscillation modes as a function of the frequency $w / 2 \pi$ parametrized by $\beta$ a in the case without external mirror and $\mathbf{b}$ in the presence of the external mirror. The solid lines start at $\beta=0$ and proceed to the right as $\beta$ grows. Both curves were computed with $\varepsilon=1 / T=1 / 1710$ and $\alpha=5$ a



b

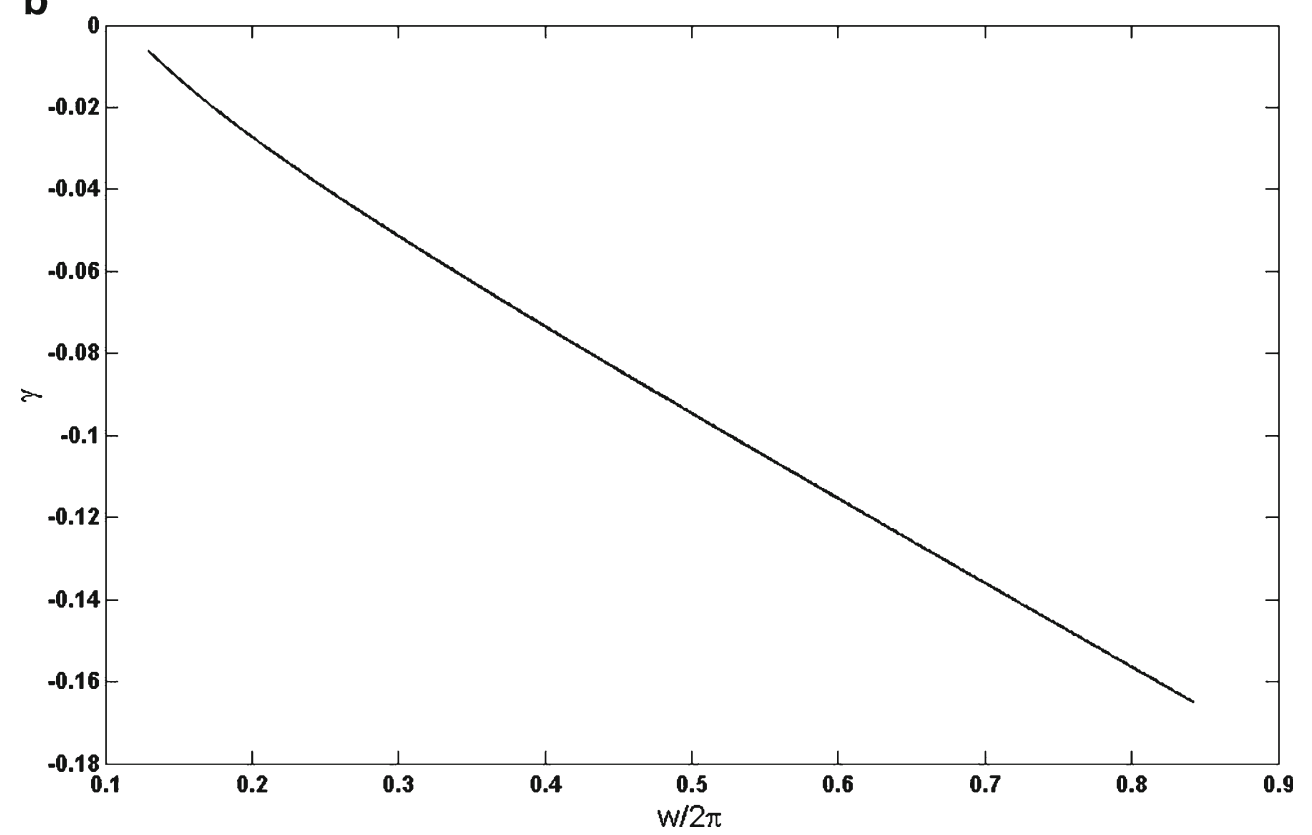


Fig. 3 Spectra of the injection amplitude $\left|E_{\mathrm{m}}\right|^{2}$ as functions of the frequency $w / 2 \pi$ for $\varepsilon=1 / T=$ $1 / 1710$ and $\alpha=5$. The feedback strengths are a $k=0, \mathbf{b} k=0.09$, and $\mathbf{c} k=0.115$. Curves $I-I I I$ correspond to $\beta=0.04,0.08$, and 0.12 , respectively
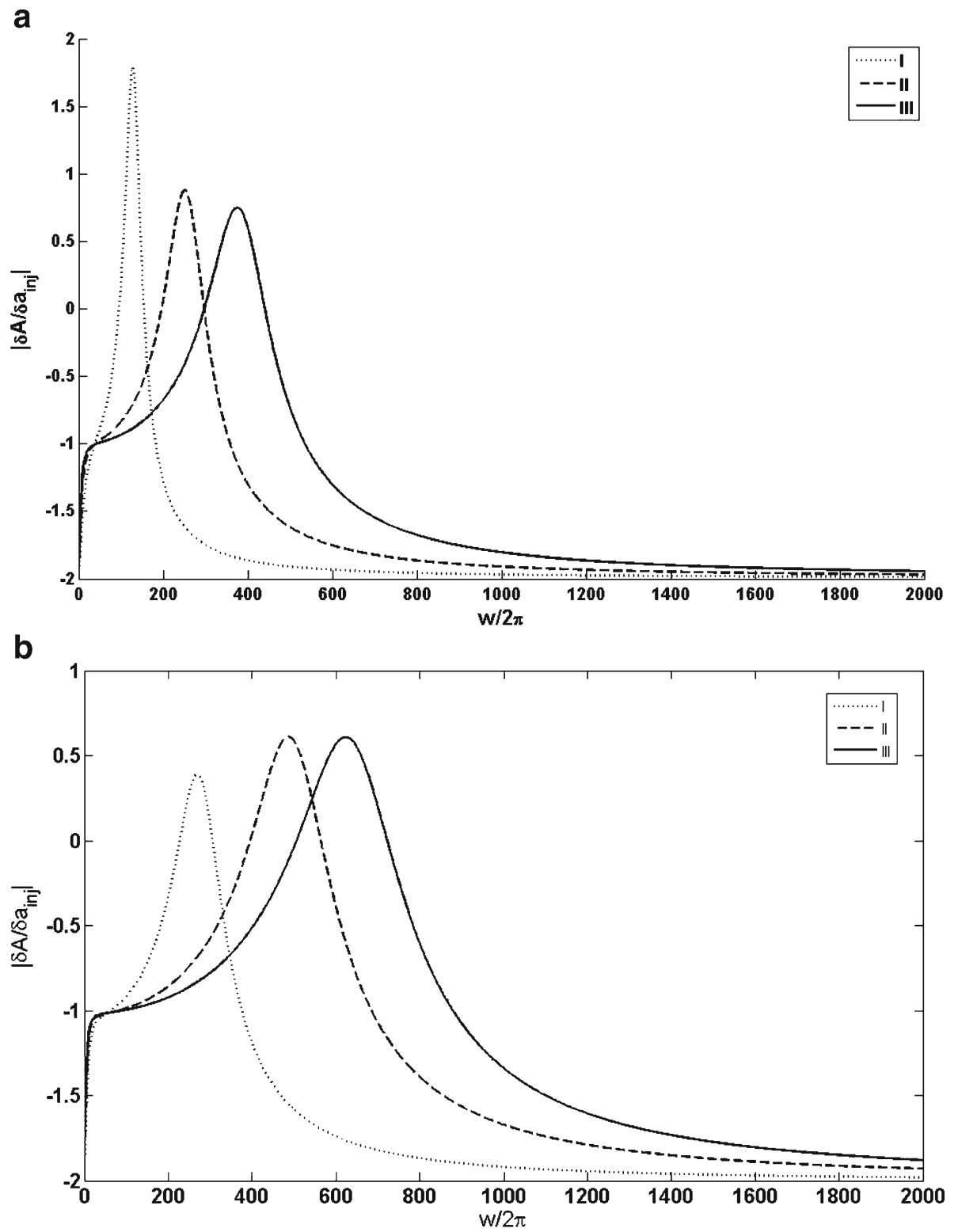

C

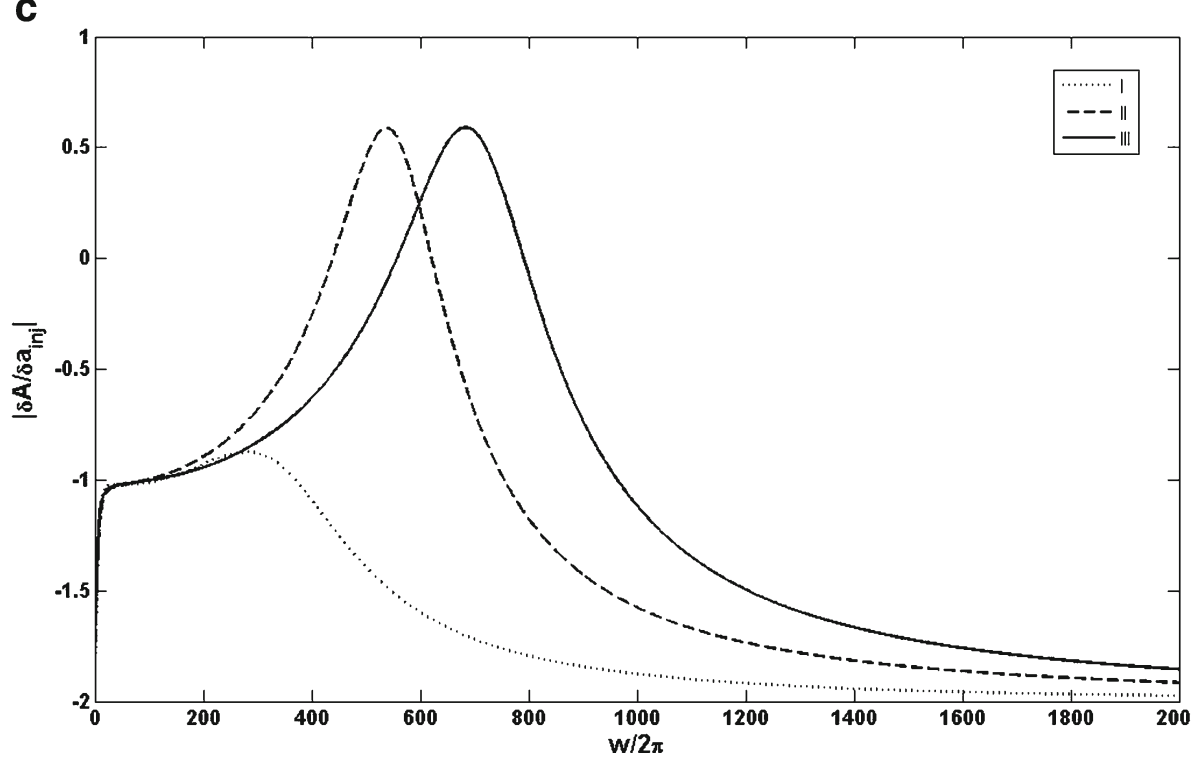


Because of the limitations of linear stability analysis, the laser-driven response has to be independently divided into injection amplitude and phase.

The injection response can therefore be expressed in the form $\delta A / \delta a_{\text {inj }}$ for $\delta \phi_{\text {inj }}=0$ and $\delta \phi / \delta \phi_{\text {inj }}$ for $\delta a_{\text {inj }}=0$.
We let $\lambda=i w$ to find the frequency response in each case. From Eqs. (13), (14), and (15), we obtain the expressions

$$
\frac{\delta A}{\delta a_{d r v}}=\frac{1}{D(i w)}\left[\begin{array}{c}
\left(\left(1+\alpha^{2}\right) N_{s t}^{2} T^{-1}\left(1+2 A_{s t}^{2}\right)+T^{-1}\left(1+2 A_{s t}^{2}\right) k N_{s t}+w^{2}\left(N_{s t}+k\right)\right) \\
+i w\left(\left(1+\alpha^{2}\right) N_{s t}^{2}-T^{-1}\left(1+2 A_{s t}^{2}\right)\left(N_{s t}+k\right)+N_{s t} k\right)
\end{array}\right]
$$

and

$$
\frac{\delta \phi}{\delta \phi_{\mathrm{drv}}}=-\frac{1}{D(i w)}\left[\begin{array}{c}
\left(\left(1+\alpha^{2}\right) N_{\mathrm{st}}^{2} T^{-1}\left(1+2 A_{\mathrm{st}}^{2}\right)-2 T^{-1}\left(1+2 N_{\mathrm{st}}\right) A_{\mathrm{st}}^{2}\left(k+N_{\mathrm{st}}\right)\right) \\
\left.+w^{2}\left(N_{\mathrm{st}}+k\right)\right)+T^{-1} N_{\mathrm{st}} k\left(1+2 A_{\mathrm{st}}^{2}\right)-2 \alpha N_{\mathrm{st}} T^{-1}\left(1+2 N_{\mathrm{st}}\right) A_{\mathrm{st}}^{2} \\
+i w\left(\left(1+\alpha^{2}\right) N_{\mathrm{st}}^{2}-T^{-1}\left(1+2 A_{\mathrm{st}}^{2}\right)\left(N_{\mathrm{st}}+k\right)+N_{\mathrm{st}} k\right)
\end{array}\right]
$$

\section{Numerical Analysis}

Figure 2 shows the damping rate $\gamma$, of the SL, in terms of the damped-oscillation angular frequency $w / 2 \pi$. The rate is obtained from Eq. (12) for different injection rates $\beta$. First, we assume the slave laser to be without an $\mathrm{EM}$, i.e., with $k=0$. In this case, Fig. 2a shows that, for $\beta=0$, the linear mode of the SL is the same as the relaxation oscillations of the solitary semiconductor laser and hence exhibits a damped oscillation, with negative $\gamma$, at a frequency of about $0.03 \mathrm{GHz}$. For weak injection rates $(\beta<0.05)$, the SL oscillates in unstable modes $(\gamma>0)$, which shows that, by itself, optical injection makes the laser unstable. In fact, injected lasers in this region are known to exhibit bifurcation routes to chaos [17]. However, the instability is reduced as $\beta$ grows, and for $\beta>0.05$, the SL oscillations become stable, with negative damping rate.

In fact, in a SL with a short external cavity, the EM affects directly the stability of the laser dynamics. The dynamical behavior of the ML as a function of $k$ (with respect to the control parameter $C_{\mathrm{p}}$ ) can be divided into two domains: low and high feedback strengths.

For very small $k$, over the complete domain $-\pi \leq C_{\mathrm{p}} \leq+\pi$, the dynamics of the laser is unstable. For increasing $k$, the range of this instability is reduced to a small domain and a new dynamics with periodic and quasi-periodic modes emerges [17]. For moderately small $k$, the region of chaos and instability is only partially removed and covers a specific $C_{\mathrm{p}}$ range.

Finally, for high feedback strengths, the chaotic dynamics is expected to disappear and the output of SL, to contain regular pulse-package, periodic, and quasi-periodic behaviors [17]. The stable-mode regime corresponds to the injection- locking regime at strong injection levels. Additional increase in the injection rate raises both the damping rate and the oscillation frequency of the linear mode. The mode transition corresponds to a broadening of the injected laser bandwidth.

Figure $2 b$ describes a SL coupled to an EM with feedback strength $k$. The plot depicts a linear mode of the injected laser in the phase space $(\gamma, w / 2 \pi)$ for feedback strength $k=0.0209$ and different injection rates $\beta$. Comparison between Fig. $2 \mathrm{~b}$ and a shows that the EM eliminates the instability domain $(0.03<w / 2 \pi<0.13)$ at weak injection rates $(\beta<0.05)$, so that the unstable mode becomes stable, with $\gamma<0$. In fact, for $k=$ 0.0209 , the operation point of the SL occurs at the frequency $w / 2 \pi=0.112$. At high injection rates, the EM can make the bandwidth of the injected laser greater than the bandwidth of the chaotic driving signal generated by the ML.

Next, with help of Eqs. 16 and 17), we have investigated the changes of the amplitude and phase of the SL under the influence of the external mirror. Figure $3 \mathrm{a}-\mathrm{c}$ displays the spectra of the injection amplitude $\delta A / \delta a_{\text {inj }}$ for three selected feedback strengths: $k=0,0.09$, and 0.115 , respectively. The

Table 1 The coordinates of the resonance peaks of the injection amplitude for different feedback strengths of external mirror and different injection rates

\begin{tabular}{llll}
\hline$k$ & $\beta$ & & \\
\cline { 2 - 4 } & 0.04 & 0.08 & 0.12 \\
\hline 0 & $(2.29,128)$ & $(1.375,349)$ & $(1.249,376)$ \\
0.09 & $(0.88,270)$ & $(1.11,487)$ & $(1.107,620)$ \\
0.115 & $(0.49,374)$ & $(1.067,591)$ & $(1.062,769)$ \\
\hline
\end{tabular}


Fig. 4 Spectra of the injection phase $\delta \phi / \delta \phi_{\text {inj }}$ as functions of the frequency $w / 2 \pi$ for $\varepsilon=1 / T=1 /$ 1710 and $\alpha=5$. The feedback strengths are a $k=0, \mathbf{b} k=0.09$, and $\mathbf{c} k=0.115$. Curves $I-I I I$ correspond to $\beta=0.04,0.08$, and 0.12 , respectively

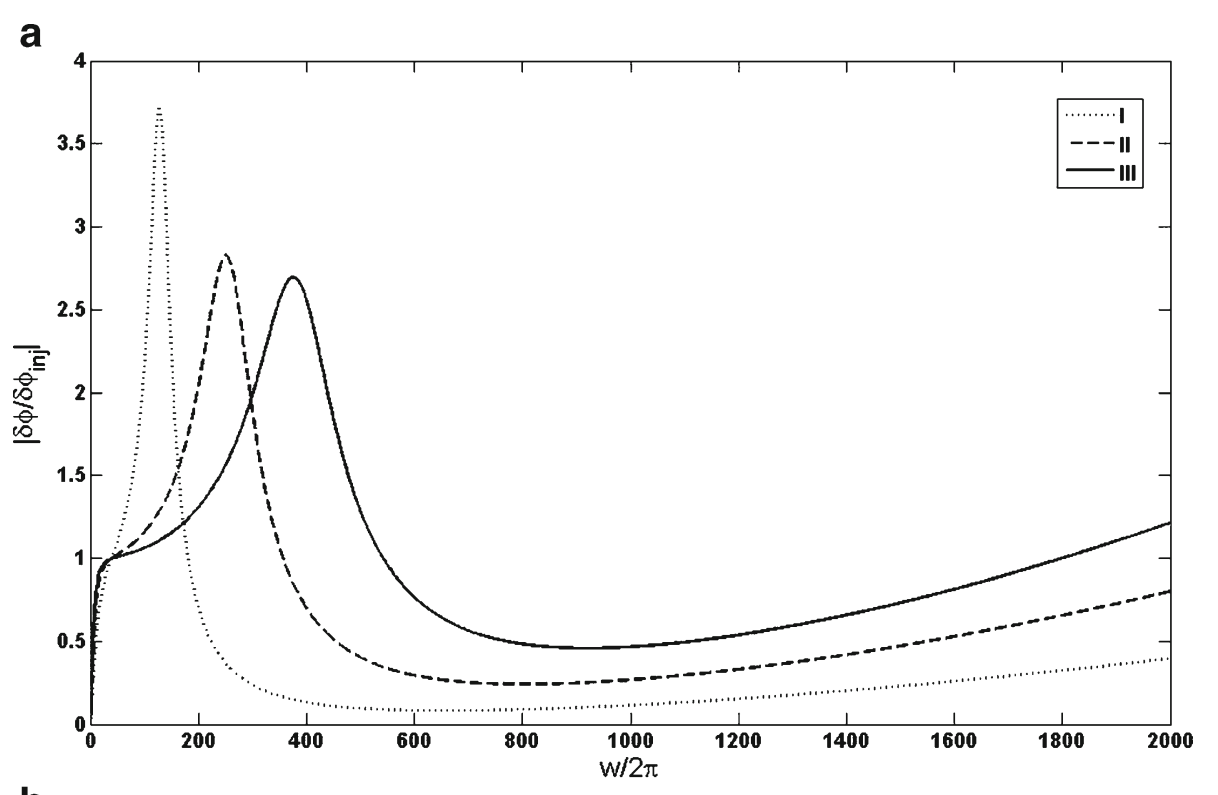

b

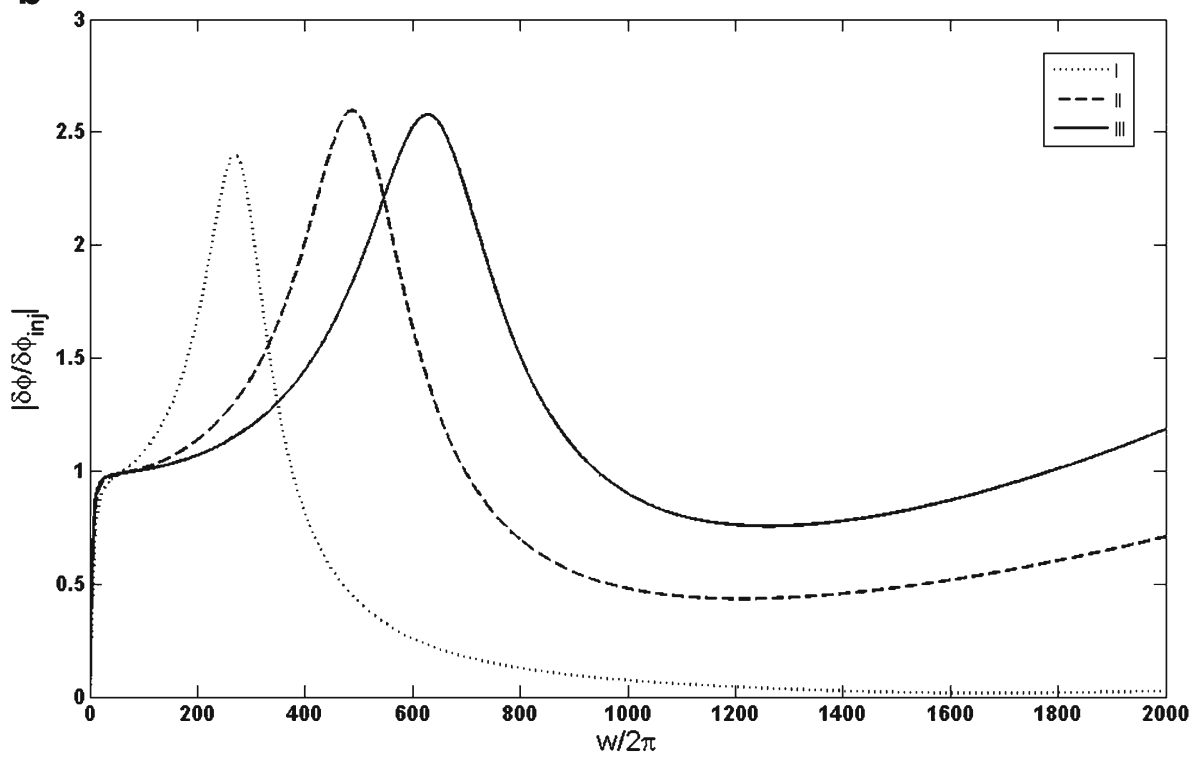

C

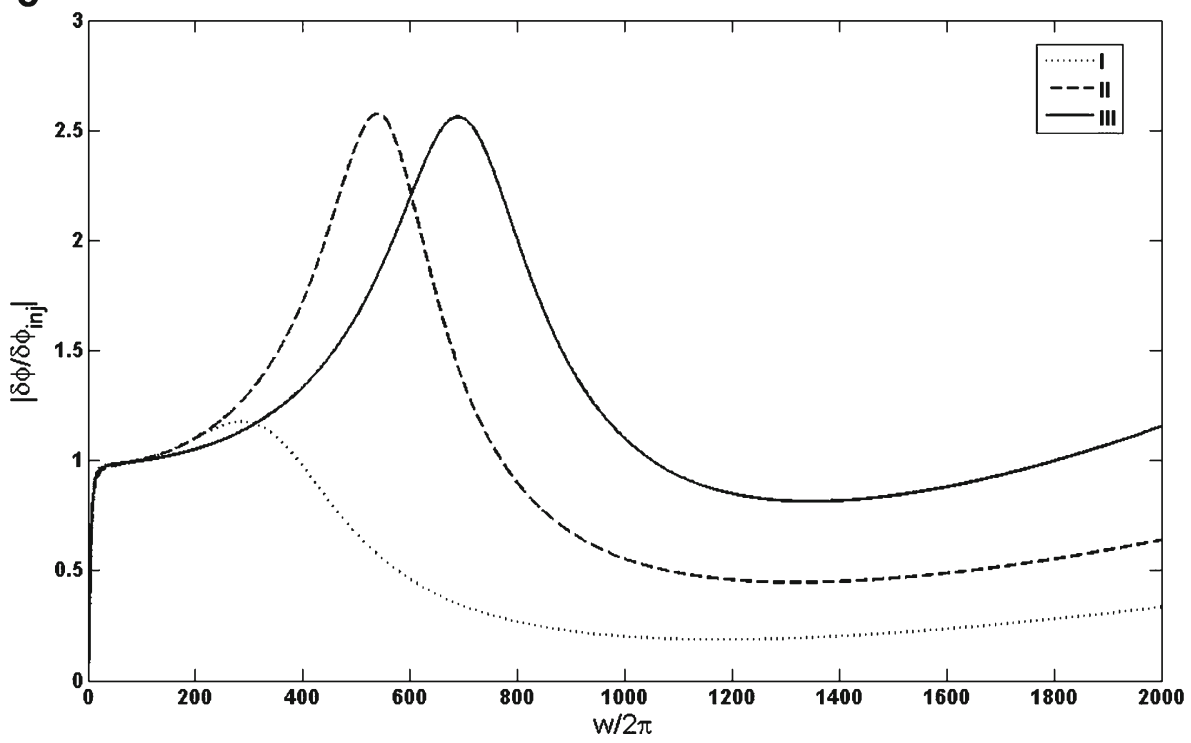


spectra of $\delta A / \delta a_{\text {inj }}$ for $\beta=0.04,0.08$, and 0.12 are labeled I, II, and III, respectively. The coordinates $\left(\delta A / \delta a_{\mathrm{inj}}, w / 2 \pi\right)$ of the resonance peaks of the injection amplitude for different injection rates are collected in Table 1.

The data in Table 1 show that, at fixed injection rate, the amplitude of the resonance peak is reduced and its frequency blue-shifted as the feedback strength grows. By contrast, different results are found if we raise the injection rate at fixed feedback. For $k=0$, the injection amplitude of the resonance peak is reduced, and its frequency blue-shifted as the injection rate increases. But for $k \neq 0$, this behavior for the injection amplitude of the resonance peak is reversed. In other words, the amplitude first grows and then remains nearly fixed as the injection rate is increased. In addition, and this is a common characteristic of Fig. 3a-c, the spectra are broadened as the feedback is strengthened. Finally, the spectrum becomes almost flat, with suppression of the resonance peak, for $\beta=0.04$ and $k=0.115$.

Next, we have investigated the effect of the EM on the phase drive of the optically injected SL. Figure $4 \mathrm{a}-\mathrm{c}$ shows the spectra $\delta \phi / \delta \phi_{\text {inj }}$ of the injection phase of the SL computed with Eq. (17) for three different selected feedback strengths, $k=0,0.09$, and 0.115 , respectively. The spectra of the injection phase for $\beta=0.04,0.08$, and 0.12 are labeled I, II, and III, respectively. The coordinates of resonance peaks of the injection phase for different feedback strengths and different injection rates are collected in Table 2.

Similar tendencies are observed for the injection amplitudes in Fig. 3 and the phases in Fig. 4. The same similarity is found for the results in Table 2, which show reduced injection phases and blue-shifted peak frequencies as the feedback strength of the EM increases. On the other hand, the spectrum structure becomes almost flat for $\beta=0.04$ and $k=0.115$. In the spectrum of the injection phase, we have found a resonance peak corresponding to the damped oscillation mode in Fig. 2. As shown in Figs. 3 and 4, the spectral bandwidth of the injection amplitude and injection phase of the injected laser grow with the injection rate and the feedback strength of the EM. The suppression of the resonance is due to the enhanced damping of the linear mode, as shown in Fig. 2. There are differences between the spectra of the injection amplitude and injection phase in Figs. 3 and 4. For the amplitude, the gain in the low-frequency region is reduced, but not for the injection phase. On the other hand, in the high

Table 2 Coordinates of phase-drive resonance peaks for different feedback strengths of the external mirror and for different injection rates

\begin{tabular}{llll}
\hline$k$ & $\beta$ & & \\
\cline { 2 - 4 } & 0.04 & 0.08 & 0.12 \\
\hline 0 & $(3.72,138)$ & $(2.82,250)$ & $(2.69,376)$ \\
0.09 & $(2.4,268)$ & $(2.59,487)$ & $(2.57,625)$ \\
0.115 & $(1.062,365)$ & $(2.55,600)$ & $(2.54,773)$ \\
\hline
\end{tabular}

frequency region, the gain for the injection phase starts to increase, but not for the injection amplitude. As evident in Figs. 3 and 4, the gain of the injection amplitude tends to -2 as $w / 2 \pi$ tends to zero, while the gain of the injection phase approaches zero as $w / 2 \pi$ tends to zero.

\section{Dynamics of the Output Intensity $\left|E_{\mathrm{s}}\right|^{2}$ of the Slave Laser}

Reference [17] showed that, for $\beta>\eta=k$, the dynamics of the SL is synchronized to the ML. In this section, choosing $\beta=$ 0.155 , we have investigated the effect of the EM on the dynamical synchronization of SL, by studying the bifurcation and time-series curves. To this end, we have fixed the feedback of the ML at $\eta=0.0455$, for which value the dynamical behavior of the ML has already been reported in [17].

We have also shown that the dynamical behavior of the SL for $\beta>\eta$ is synchronized with the ML in the $-\pi<C_{\mathrm{p}}<\pi$ range. Figure 5a shows $\left|E_{\mathrm{s}}\right|^{2}$ as a function of $C_{\mathrm{p}}$, which displays chaotic $(\mathrm{CH})$, quasiperiodic $(\mathrm{QP})$, period-7 (P7), period-1 (P1), and then chaotic dynamics. As shown in Fig. $5 b$, for an EM with $k=0.0455$, the SL dynamics is synchronized to the ML, but both the amplitude and range of instability are smaller. The instability of the SL increases as $k$ rises to 0.115 , as shown in Fig. 5 c. As also shown, the $\mathrm{P} 7$ and $\mathrm{P} 1$ dynamics have been eliminated, replaced by QP dynamics. As a result, in the $k<\eta$ range, the SL synchronizes to the ML and the stability of the SL grows with $k$. On the other hand, in the $k>\eta$ range, the SL is out of synchronization with the ML, and its stability decreases.

The time evolutions of $\left|E_{\mathrm{s}}\right|^{2}$ for $k=0, k=0.09(<\eta)$ and $k=$ $0.115(>\eta)$ are plotted in Fig. 6a-c, respectively. Clearly, the external mirror affects the output of the SL.

To validate the above results, we have investigated the time series of $\left|E_{\mathrm{s}}\right|^{2}$. To this end, we chose a feedback strength $\eta=$ 0.0455 for the ML, so that the dynamics of $\left|E_{\mathrm{m}}\right|^{2}$ is chaotic [17]. The time evolution of $\left|E_{\mathrm{s}}\right|^{2}$, with $\beta=0155$, is presented in Fig. 6a-c for $k=0, k<\eta$, and $k>\eta$, respectively.

The time series in Fig. 6a represents chaotic dynamics for $\left|E_{\mathrm{s}}\right|^{2}$ and $\delta A / \delta a_{\text {inj }}$, the dynamics of $\left|E_{\mathrm{s}}\right|^{2}$ being synchronized with $\left|E_{\mathrm{m}}\right|^{2}$. Figure $6 \mathrm{~b}$ also shows synchronized chaotic dynamics between $\left|E_{\mathrm{s}}\right|^{2}$ and $\left|E_{\mathrm{m}}\right|^{2}$, but the amplitude of $\left|E_{\mathrm{s}}\right|^{2}$ is reduced. The chaotic dynamics of $\left|E_{\mathrm{s}}\right|^{2}$ loses synchronization with $\left|E_{\mathrm{m}}\right|^{2}$ as the feedback strength of the EM of the SL is increased to $k=0.115$, as shown by Fig. $6 \mathrm{c}$.

When $\beta$ exceeds $\eta$, in the $0<k<\eta$ range, $\left|E_{\mathrm{s}}\right|^{2}$ synchronizes with $\left|E_{\mathrm{m}}\right|^{2}$. In fact, in this range the amplitude of $\left|E_{\mathrm{s}}\right|^{2}$ decreases as $k$ increases, and the growth of $k$ is not effective on the synchronization of $\left|E_{\mathrm{s}}\right|^{2}$ and $\left|E_{\mathrm{m}}\right|^{2}$. By contrast, in the $k>\eta$ range, not only is the synchronization between $\left|E_{\mathrm{s}}\right|^{2}$ and $\left|E_{\mathrm{m}}\right|^{2}$ lost, but also the instability of $\left|E_{\mathrm{s}}\right|^{2}$ grows with $k$, as indicated by the evolution of the height of the peak following the highest maximum. 
Fig. 5 Bifurcation diagrams of the SL intensity $\left|E_{\mathrm{s}}\right|^{2}$ as a function of the feedback phase $C_{\mathrm{p}}(-\pi<$ $\left.C_{\mathrm{p}}<\pi\right)$, with $\beta=0.155$ for a $k=0$, b $k=0.0455=\eta$, and $\mathbf{c} k=$ $0.115>\eta$ a

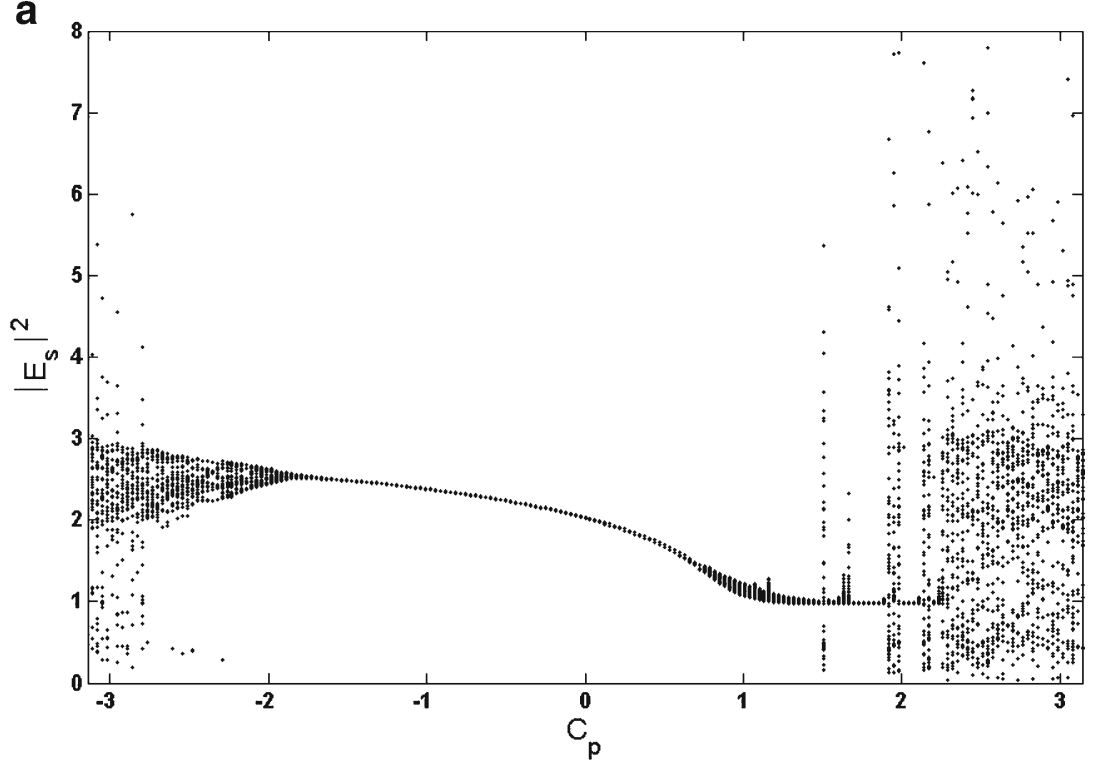

b

Fig. 6 Time series of $\left|E_{\mathrm{m}}\right|^{2}$, with $\beta=0.155$ for $\mathbf{a} k=0, \mathbf{b} k=$

$0.0455=\eta$, and $\mathbf{c} k=0.115>\eta$ a

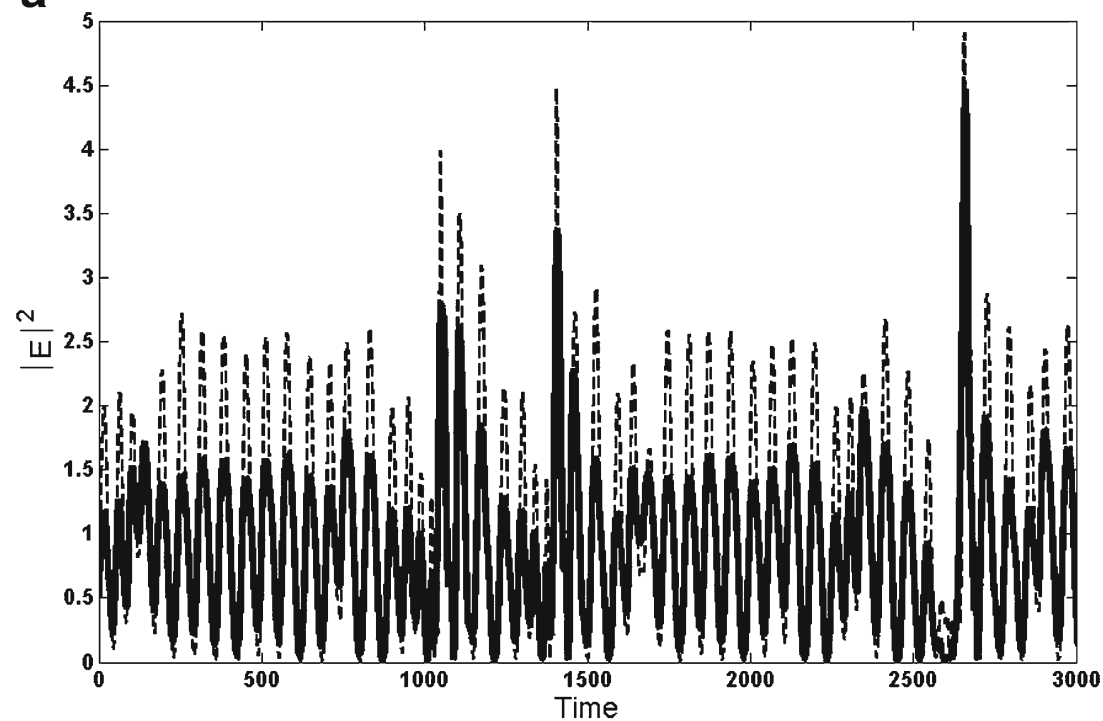

b
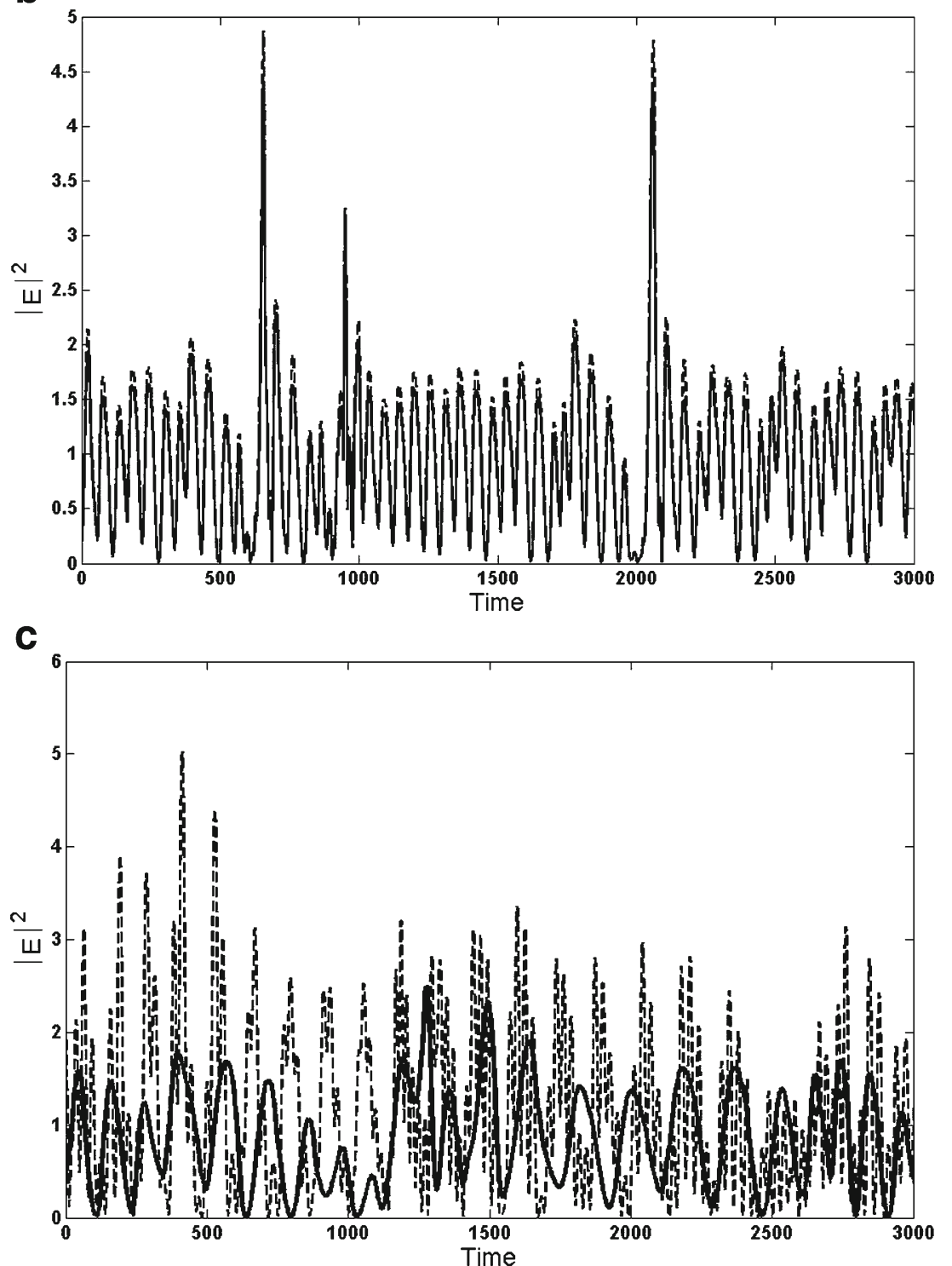


\section{Conclusion}

We have studied feedback-induced chaos from a master semiconductor laser into a slave laser coupled to an external mirror. We have carried out linear stability analysis for rate equations and analytically investigated the bandwidth of optically driven semiconductor lasers.

The main conclusions of this paper are summarized as follows: as suggested by [19], the linear mode of the injected laser moving in the phase space $(\gamma, w)$ shows unstable behavior in the frequency range of $(0.03<w<0.13)$, which becomes stable when the external mirror is added to the injected-laser system. We have also shown that, under the effect of the external mirror, the spectral bandwidth of the injection amplitude and injection phase of the injected laser increase as the injection rate and the feedback strength of the external mirror grows. In fact, the response of the slave mirror can be broader and more enhanced than that of the broadband chaotic injecting signal from the master laser. We have shown that the resonance suppression is due to the enhanced damping of the linear mode. Our numerical simulations verified that, subject to strong injection and under the influence of the external mirror, the slave laser can exhibit a linear-like driven response to the master laser. We notice that the gain for the two spectra are different at low or at high frequencies; the injection phase of the injected laser is therefore more likely to respond linearly than the injection amplitude.

The bifurcation and time-series curves have shown that the external mirrors can benefit the stability of semiconductor lasers. We have found that an appropriate ratio between the feedback strengths of the slave laser and of the master laser synchronizes their outputs. We had shown beforehand that, for $\beta>\eta=k$, the outputs are synchronized. In this paper, for $\eta=$ 0.0455 and $\beta=0.155$, we have seen that, for $0<k<\eta$, the oscillation modes of the slave laser are synchronized with the modes of the master laser, although the amplitude and instability are reduced. The slave laser becomes more unstable as $k$ increases to 0.115 , while the synchronization between the outputs of two lasers is lost.

\section{References}

1. J. Wang, M.K. Haldar, L. Li, F.V.C. Mendis, IEEE Photon. Technol. Lett 8, 34-36 (1996)

2. T.B. Simpson, J.M. Liu, IEEE J. Quantum Electron. 32, 1456-1468 (1996)

3. A. Murakami, K. Kawashima, K. Atsuki, IEEE J. Quantum Electron. 39, 1196-1204 (2003)

4. W. Jia-Gui, X. Guang-Qiong, C. Liang-Ping, W. Zheng-Mao, Opt. Commun. 282, 3153-3156 (2009)

5. S. Banerjee, L. Rondoni, S. Mukhopadhyay, A.P. Misra, Opt. Commun. 284, 2278-2291 (2011)

6. S. Banerjee, L. Rondoni, S. Mukhopadhyay, Opt. Commun. 284, 4623-4634 (2011)

7. A. Jafari, K. Mabhouti, S. Afrang, A. Siahcheshm, Opt. Laser Technol. 44, 1398-1405 (2012)

8. L. Pecora, T.L. Carroll, Phys. Rev. Lett. 64, 821-824 (1990)

9. I. Fischer, Y. Liu, P. Davis, Phys. Rev. Lett. 62, 011801R-011804R (2000)

10. K. Kusumoto, J. Ohtsubo, Opt. Lett. 27, 989-991 (2002)

11. T. Heil, I. Fischer, W. Elsaber, Phys. Rev. A 58, R2672-R2675 (1998)

12. G.H.M. van Tartwijk, A.M. Levine, D. Lenstra, IEEE J. Sel. Top. Quant. Electron. 1, 466 (1995)

13. J.M. Buldu', J. Garci'a-Ojalvo, M.C. Torrent, IEEE J. Quantum Electron. 40, 647-650 (2004)

14. A. Argyris et al., Nature 438, 343 (2005)

15. C.R. Mirasso, R. Vicente, P. Colet, J. Mulet, T. Pe'rez, CR Phys. 5, 613 (2004)

16. T.B. Simpson, J.M. Liu, A. Gavrielides, IEEE Photon. Technol. Lett. 7, 709-711 (1995)

17. A. Jafari, H. Sedghi, K.H. Mabhouti, S. Behnia, Opt. Commun. 284, 3018-3030 (2011)

18. A. Murakami, Phys. Rev. E 65, 056617-056625 (2002)

19. T. Heil, I. Fischer, W. ElsaBer, Phys. Rev. Lett. 87, 243901-2439015 (2001)

20. T. Heil, I. Fischer, W. ElsaBer, B. Krauskopf, K. Green, A. Gavrielides, Phys. Rev. E 67, $066214-066224$ (2003)

21. F. Mogensen, H. Olesen, G. Jacobsen, IEEE J. Quantum Electron. QE 21, 784-793 (1985)

22. P.M. Alsing, V. Kovanis, A. Gavrielides, T. Erneux, Phys. Rev. A 53, 4429-4434 (1996)

23. J. Ohtsubo, Chaos synchronization in semiconductor lasers. Semiconductor lasers stability, Instability and chaos (Springer, Atlanta, 1965)

24. Y. Liu, N. Kikuchi, J. Ohtsubo, Phys. Rev. E 51, R2697-R2700 (1995)

25. A. Murakami, J. Ohtsubo, IEEE J. Quantum Electron. 34, 1979$1986(1998)$ 